\title{
Mercado Laboral de las Personas Discapacitadas en España
}

\author{
Ricardo Pagán Rodrigez * \\ Andrés.J. Marchante Mera **
}

RESUMEN

En este trabajo, partiendo de un análisis inicial basado en el estudio de las politicas públicas dirigidas a las personas discapacitadas y de los problemas en la definición de la discapacidad, se analizan las características sociodemográficas de las personas discapacitadas en edad de trabajan y aquellas que están trabajando. A partir de la información disponible en el Panel de Hogares de la Unión Europea para el año 1998, los resultados obtenidos permiten afirmar que uno de los principales obstáculos para la integración laboral de las personas discapacitadas se situa en la primera fase del proceso de inserción, es decir, en pasar de la inactividad a la actividad. Estos resultados tienen implicaciones en las futuras politicas públicas dirigidas a favorecer la integración laboral y social de este colectivo.

JEL Clasificación: I1, J2 y J3.

Palabras Claves:

Discapacidad, empleo y participación laboral.

- Departamento de Economía Aplicada. Universidad de Mâlaga • rpr@uma.es

** Departamento de Economia Aplicada. Universidad de Málaga • marchante@uma.es

Los autores agradecen la ayuda financiera recibida del Instituto de Estudios Fiscales para la realización de esta parte del estudio (contrato $n^{\circ}$ 806/16.2148). 
ABstract

In this piece of work, beginning with a preliminary analysis of the public policies for disabled persons and the problems associated to the concept of disability, we analyse the sociodemographics characteristics of working age and employed disabled persons in Spain. Using the data from the European Housebold Panel for 1998, our results sbow that the main barrier for the labour integration of the disabled person is found in the first phase of the process, this is to say, from the inactivity to labour activity. These results bave implication for the future public policies lead to this specific group.

JEL Classification: $\mathrm{I} 1, \mathrm{~J} 2$ y $\mathrm{J} 3$.

KEY WORDS:

Disability, employment, labour participation.

\section{INTRODUCCIÓN}

Desde la entrada en vigor del Tratado de Ámsterdam en 1997, que incluía una cláusula de no discriminación a favor de los minusválidos (artículo 13), hasta las últi-

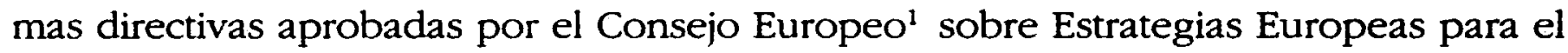
Empleo, todas las acciones y medidas a favor de la igualdad de oportunidades de las personas discapacitadas poseen un carácter prioritario. Incluso, el año 2003 ha sido oficialmente declarado como Año Europeo de las Personas Discapacitadas. La mayoría de los Estados miembros han ampliado la gama de medidas legales, financieras y voluntarias y los servicios para aplicarlas. La proliferación y diversificación creciente de estrategias encaminadas a fomentar las oportunidades de empleo de las personas con discapacidad constituye un cambio significativo y está favoreciendo, sin duda, el acceso al empleo de grupos anteriormente marginados en el mercado laboral.

Las personas discapacitadas encuentran mayores dificultades para encontrar un empleo que el resto de la población debido a que se enfrentan a unas necesidades y dificultades especiales a la hora de poder acceder y permanecer en el mercado de trabajo. Estas necesidades y dificultades tienen su reflejo en una mayor tasa de desempleo para las personas discapacitadas en comparación con la estimada para toda la población. El alto nivel de desempleo general, los prejuicios sociales y empresariales, la falta de adaptación de los puestos de trabajo y la baja cualificación

1. En el Consejo Europeo de Luxemburgo de 1997 uno de los objetivos de las políticas contra el desempleo en los Estados miembros era la mejora de la capacidad de inserción profesional. Una de las conclusiones se refería específicamente a las personas con discapacidad: "Los Estados miembros prestarán especial atenciōn a las dificultades que puedan experimentar las personas minusválidas para incorporarse a la vida activa. (punto 79 de las Conclusiones del Consejo). 
profesional inciden negativamente en la inserción laboral de las personas con discapacidad. Existen barreras a la contratación, más sutiles que las arquitectónicas o las de comunicación, que impiden la aplicación efectiva del principio de igualdad de oportunidades en materia de empleo a las personas con discapacidad y las hacen dependientes de las políticas de protección.

El interés entre los investigadores por la discapacidad desde un punto de vista económico ha aumentado en los últimos años debido principalmente a tres factores (Salkever y Sorkin, 2003): a) la aparición de nuevas bases de datos disponibles que tratan la discapacidad y problemas de salud desde un óptica específica; b) la aparición de nueva legislación antidiscriminatoria y dirigida a conseguir la plena igualdad de oportunidades en todos los ámbitos de la vida, especialmente en el mercado laboral; y c) el incremento en los costes asociados a la discapacidad desde el punto de vista del gasto público.

La literatura existente sobre discapacidad es relativamente reciente, siendo el trabajo de Berkowitz y Johnson (1974) uno de las primeras contribuciones en el análisis de la discapacidad. A nivel internacional abundan los estudios relacionados con la discapacidad (por ejemplo, Jenkins y Rigg, 2003; Kidd et al., 2000; O`Donnell, 1998; Bardasi et al., 2000; Burchardt, 2000; Baldwin y Johnson, 1994). Sin embargo, la existencia de estudios sobre la situación laboral y profesional de las personas discapacitadas en el mercado de trabajo español es escasa. Por ejemplo, cabe destacar los trabajos realizados por el Consejo Económico y Social (1995) y más recientemente, los realizados por Malo y García (2001) y por el Grupo de Expertos Europeos. Sin embargo, la mayoría de avances y logros sobre el problema de la integración laboral de las personas discapacitadas lo hacen desde el mundo del derecho ${ }^{2}$.

2. Existe un amplia cantidad de textos normativos aprobados en las dos últimas décadas que desarrollan la Ley de Integración Social de Minusvâlidos (LISMI) de 1982, y abordan aspectos relacionados con la formación, empleo selectivo, incentivos al empleo estable, cuota de reserva en las empresas con más de 50 trabajadores, empleo protegido, etc. Véase por ejemplo:

- ORDEN de 24 de julio de 2000 (B.O.E. núm. 190 de 9-08-2000) por la que se regula el procedimiento administrativo referente a las medidas alternativas de carácter excepcional al cumplimiento de la cuota de reserva del 2 por 100 en favor de trabajadores discapacitados en empresas de 50 o más trabajadores, reguladas por el Real Decreto 27/2000, de 14 de enero.

- REAI DECRETO 27/2000, de 14 de enero, (B.O.E. núm. 22 de 26-01-2000), por el que se establecen medidas alternativas de carácter excepcional al cumplimiento de la cuota de reserva del 2 por 100 en favor de trabajadores discapacitados en empresas de 50 o más trabajadores.

- REAL DECRETO 4/1999, de 8 de enero (B.O.E. 26-01-1999), por el que se modifica el artículo 7 del Real Decreto 1451/1983, de 11 de mayo, por el que, en cumplimiento de lo previsto en la Ley 13/ 1982, de 7 de abril, se regula el empleo selectivo y las medidas de fomento del empleo de los trabajadores minusválidos.

- ORDEN de 16 de octubre de 1998 (B.O.E. 21-11-1998), por la que se establecen las bases reguladoras para la concesión de las ayudas y subvenciones públicas destinadas al fomento de la integración laboral de los minusválidos en centros especiales de empleo y trabajo autónomo. 
Los objetivos del trabajo son varios. En primer lugar, estudiar los principales pilares en los que se sustentan las políticas públicas dirigidas al colectivo formado por las personas discapacitadas. En segundo lugar, analizar la problemática relativa a la definición del concepto de discapacidad. Y en tercer lugar, presentar datos más actuales sobre las características socioeconómicas y laborales de la población discapacitada en edad de trabajar y la que está actualmente empleada en España. La estructura del trabajo sigue estos objetivos apuntados, además de incluir en el último apartado las principales conclusiones del trabajo.

2. Discapacidad: POLÍtTCAS PÚBlicas, CONCEPTO Y SITUACIÓN ACTUAL

\subsection{Políticas públicas}

A nivel europeo, todos los Estados miembros reconocen el riesgo de exclusión de las personas discapacitadas en el mercado de trabajo, como así lo reflejan las altas tasas de desempleo y los bajos niveles de formación y cualificación profesional que éstos poseen. En los distintos Planes Nacionales de Empleo de cada uno de los Estados miembros se recogen dos tipos de políticas públicas:

a) Políticas pasivas basadas principalmente en prestaciones económicas sustitutivas de los ingresos derivados del trabajo.

b) Políticas activas orientadas a incentivar la formación ocupacional de la persona discapacitada y su inserción laboral.

Algunos Estados miembros como Suecia y Holanda incorporan como objetivo nacional el incremento del nivel de inclusión social de las personas discapacitadas, mientras que en otros países, entre ellos España, sólo se incluyen objetivos específicos para el fomento de la integración laboral de las personas discapacitadas.

La influencia de las políticas y directrices europeas en los temas relacionados con la integración social y laboral de las personas discapacitadas ha tenido un efecto y reflejo inmediato en los diferentes Planes Nacionales de Empleo de los Estados miembros, especialmente después de la cumbre de Luxemburgo celebrada en noviembre de 1997. En los Planes de Empleo del Reino de España se recogen directrices específicas que afectan a la integración de las personas discapacitadas y que se han visto con el tiempo complementadas por nuevas acciones y objetivos. También, los programas de acción comunitaria HELIO y la iniciativa EMPLEO-HORIZON han jugado un papel muy relevante en la integración real de este cölectivo ya que ha contribuido a mejorar la concienciación de los agentes intermedios hacia ellos (funcionarios públicos, profesionales y técnicos, empresarios, sindicatos, etc.) y a la construcción de redes y asociaciones dirigidas hacia nuevos enfoques y propuestas en temas de discapacidad a nivel europeo. 
En España, las políticas sociales públicas desarrolladas en materia de empleo para las personas discapacitadas giran en torno a tres grandes ámbitos, los dos primeros encuadrados dentro de las políticas pasivas y la última dentro de las políticas activas:

\section{A) Protección económica.}

Las prestaciones económicas sustitutivas de los ingresos derivados del trabajo pueden clasificarse en función del hecho que las origina (prestaciones por incapacidad temporal, por incapacidad permanente y otras prestaciones) o en función de su naturaleza (prestaciones contributivas y no contributivas). Las cuantías mínimas y máximas se establecen según la normativa vigente, es decir, en la Ley de Presupuestos Generales del Estado de cada año. Los requisitos, duración, cuantía, etc. se encuentran recogidas en el marco legislativo que las regula así como en diferentes manuales y monografías de variada difusión que han aparecido y han sido editadas, en muchos casos, por el propio Ministerio de Trabajo y Asuntos Sociales o por las Consejerías de Asuntos Sociales de algunas Comunidades Autónomas, como, por ejemplo, la Comunidad Andaluza.

\section{B) Tratamiento fiscal de las rentas de trabajo.}

En la Ley 40/98 de 9 de diciembre, del Impuesto sobre la Renta de la Personas Físicas (IRPF) y otras normas tributarias se establecía un incremento en las deducciones aplicables a los rendimientos del trabajo para el caso de las personas discapacitadas a partir del ejercicio de 1999. También, en la nueva regulación del IRPF se contemplan deducciones para la inversión en la vivienda habitual para aquellos contribuyentes discapacitados que realicen obras e instalaciones de adecuación, así como la reducción de la base imponible por aportaciones a planes de pensiones y a mutualidades a favor de aquellas personas que tengan un grado de discapacidad superior al $65 \%$. Además, se consideran exentas del impuesto las rentas obtenidas y reconocidas por la seguridad social como consecuencia de incapacidad permanente absoluta o gran invalidez y las prestaciones familiares por hijo minusválido a cargo, entre otras.

C) Medidas para mejorar la empleabilidad, el acceso al empleo y la calidad del mismo.

En función de las capacidades de la persona discapacitada es posible identificar varias opciones en su itinerario de inserción laboral:

a) Los Centros Ocupacionales. Su finalidad es asegurar los servicios de terapia ocupacional y de ajuste personal a los discapacitados cuando por el grado de su minusvalía no pueden incorporarse a una empresa o Centro Especial de Empleo. 
b) Los Centros Especiales de Empleo. Tienen como objetivo principal realizar un trabajo productivo, participando regularmente en las operaciones de mercado y teniendo como finalidad el asegurar un empleo remunerado y las prestaciones de servicios de ajuste personal y social que requieran los trabajadores minusválidos, a la vez que han de ser un instrumento de integración del mayor número de personas discapacitadas al mercado laboral ordinario. Es decir, se configura como una etapa transitoria por la que pasa el trabajador hasta su posterior integración en el mercado laboral no protegido.

c) El Empleo de Apoyo. Es el empleo integrado en la comunidad dentro de empresas normalizadas, para aquellas personas con discapacidad que tradicionalmente no han tenido posibilidad de acceso al mercado laboral, mediante la provisión de los apoyos necesarios dentro y fuera del lugar de trabajo y en condiciones de empleo lo más similares posible en trabajo y sueldo a las de otro trabajador sin discapacidad en un puesto equiparable dentro de la misma empresa.

d) El Empleo Normalizado u Ordinario. Es el empleo en empresas normalizadas en las que la gran mayoría de los trabajadores no tienen ningún tipo de discapacidad. También entraría en esta categoría el autoempleo a través de la creación de una empresa o dándose de alta como trabajador autónomo, tenga o no otros trabajadores a su cargo.

También, para favorecer la integración laboral de las personas discapacitadas se han establecido instrumentos jurídicos de discriminación positiva dirigidos a fomentar su contratación en el sistema ordinario de trabajo, su integración como trabajadores autónomos y su incorporación a Centros Especiales de Empleo. Entre ellas se encuentran las subvenciones a la contratación de personas discapacitadas (contrato indefinido para trabajadores minusválidos, contrato temporal de minusválidos acogido a fomento del empleo, contrato para la formación de trabajadores minusválidos, contratos en prácticas de trabajadores minusválidos, integración de minusválidos en Cooperativas y Sociedades Anónimas Laborales, etc.), las medidas para la adecuación de los puestos de trabajo, la dotación de medios adecuados para la prevención de riesgos laborales específicos, y quizás las más significativas, el establecimiento de cuotas obligatorias de empleo para las personas discapacitadas y el fomento del empleo en las administraciones públicas.

Con respecto a las cuotas de reserva se establece que aquellas empresas que tengan más de 50 trabajadores fijos dentro de su plantilla están obligadas a emplear a un número de trabajadores discapacitados no inferior al $2 \%$ de la plantilla (Ley 13/82, art. 38.1). La aprobación del Real Decreto 27/2000 sobre las Medidas Alternativas planteó tres nuevas alternativas excepcionales a la obligación legal de contratación del $2 \%$ de trabajadores discapacitados sobre el total de la plantilla de la 
empresa, cuando ésta tenga más de 50 empleados. La primera y segunda vía del Real Decreto permite a la empresa la contratación de productos y/o servicios con un Centro Especial de Empleo o Trabajador Autónomo Discapacitado, mientras que la tercera vía se plantea como una donación a fundación o asociación declarada de utilidad pública. El objetivo de estas alternativas es dotar de una mayor flexibilidad a las empresas en el cumplimiento de la ley y en último extremo, asegurar la creación de empleo para las personas discapacitadas de modo directo o indirecto.

Las administraciones públicas también están sujetas a la cuota de reserva del $2 \%$, si bien se ha establecido un mecanismo para elevar dicha cuota al $3 \%$ de las vacantes en las ofertas anuales de empleo público existentes. El acceso y provisión de puestos de trabajo para las personas con discapacidad dentro de las distintas administraciones públicas se rige por cinco directrices: a) la reserva de cupo para las personas discapacitadas en las ofertas de empleo público; b) la concurrencia en igualdad de oportunidades con los demás aspirantes a las pruebas selectivas; c) la adaptación necesaria en tiempo y medios para la realización de dichas pruebas selectivas a instancia de los interesados; d) la acreditación de las condiciones personales de aptitud para el desempeño de las funciones correspondientes al puesto de trabajo y; e) la adaptación del puesto de trabajo solicitado en función de la discapacidad.

También, se están realizando esfuerzos importantes en cuanto a lo que se denomina "empleo selectivo", es decir, al conjunto de normas que regulan los derechos de aquellos trabajadores a los que le ha sobrevenido una minusvalía para la reincorporación a su antiguo puesto de trabajo, una vez terminados tos correspondientes procesos de rehabilitación y recuperación. La readmisión del trabajador le da al empresario el derecho a una rebaja en la cuota patronal de la seguridad social del $50 \%$ durante un periodo de dos años.

Otro aspecto importante está relacionado con la accesibilidad física y la ergonomía. La Ley de Prevención de Riesgos Laborales (Ley 31/95) establece como principio de acción preventiva la adaptación del puesto de trabajo a la persona tanto en lo que respecta a la concepción de los puestos como en la elección de los equipos y métodos de trabajo, y obliga al empresario a proporcionar a sus trabajadores equipos de protección individual adecuados al desempeño de sus actividades. Además, se establecen ayudas a los empresarios para la adaptación de los puestos y mejorar la calidad en el trabajo.

\subsection{CONCEPTO}

A nivel europeo, uno de los problemas principales que se presentan al analizar la situación laboral y social de las personas discapacitadas es la falta de fuentes estadísticas específicas dirigidas a este colectivo. Además, cada Estado miembro de 
la Unión Europea utiliza su propio sistema para definir la población discapacitada, lo que dificulta la realización de estudios comparativos a nivel europeo.

Es posible detectar dos problemas importantes al utilizar fuentes estadísticas de diferentes países y poder realizar estudios comparativos (Malo y García 2001). En primer lugar, la gran mayoría de las encuestas disponibles sobre discapacidad están basadas en criterios subjetivos, es decir, son los propios entrevistados los que tienen que valorar su estado de salud y discapacidad. $Y$ en segundo lugar, existen importantes diferencias conceptuales entre deficiencia, discapacidad, limitación para la realización de determinadas actividades, etc. entre las diferentes encuestas disponibles. Incluso se aprecian dificultades y problemas de traducción a diferentes idiomas de conceptos clave relacionados con la discapacidad. Según los últimos datos, esta variación en la forma de definir la población objetivo provoca que el número de personas discapacitadas existentes en Europa varie entre 17 y 37 millones de personas.

Existe poco consenso sobre cuál debe ser la definición del concepto de discapacidad $^{3}$. La Organización Internacional de la Salud (OIS) define discapacidad como "una pérdida o anormalidad en el cuerpo o en las funciones físicas, psíquicas o psicológicas". Estas deficiencias pueden llevar al individuo a una limitación en las actividades que éste desarrolla. Entre estas actividades pueden encontrarse aquellas relacionadas con su participación en el mercado laboral.

En este trabajo se utiliza una definición de discapacidad que se encuadra dentro del concepto dado por la OIS y que está basada en si una persona tiene una limitación en su estado de salud que le impida desarrollar su actividad diaria. La fuente estadística utilizada en este trabajo es el Panel de Hogares de la Unión Europea (PHOGUE) para el periodo comprendido entre los años 1995 y $1998^{4}$. El cuestionario utilizado en esta encuesta contiene preguntas relacionadas con la salud del entrevistado y es el propio entrevistado el que valora su estado de salud actual. Esto significa que la definición de persona discapacitada se basará en la propia valoración del entrevistado y no en la de los servicios de salud pública existentes.

Para identificar a una persona discapacitada se recurre a dos preguntas concretas incluidas en el cuestionario:

PH002: ¿Tiene alguna enfermedad crónica física o mental o alguna incapacidad o deficiencia crónica? Si es "SI", entonces,

PHOO3: ¿Le impide esta enfermedad crónica física o mental o esta incapacidad o deficiencia desarrollar su actividad diaria? Si, intensamente/ Si, hasta cierto punto/ No.

3 Véase el trabajo de la Universidad de Brunel (2001) sobre las diferentes definiciones de discapacidad utilizadas en Europa.

4 Aunque también está disponible el año 1994, las preguntas del cuestionario utilizadas para identificar a una persona discapacitadas no es aplicable en dicho año lo que impide su utilización en este trabajo. 
Aquellas personas que responden a la pregunta $\mathrm{PH} 003$ con un " $\mathrm{Si}$ " (intensamente o hasta cierto punto) son consideradas como discapacitadas. Esta manera de identificar a las personas discapacitadas no coincide enteramente con la definición del OIS ni con la utilizada por los servicios administrativos de la Seguridad Social. Sin embargo, esta definición ha sido utilizada en trabajos como los de Burchardt (2000), Malo y García (2001) y Jenkins y Rigg (2003), y es actualmente la utilizada por el Grupo de Expertos Europeos (European Expert Network) ${ }^{5}$ en los estudios hasta la fecha realizados sobre el empleo para las personas discapacitadas. Por ejemplo, dentro del ámbito europeo y según Malo y García (2001), los países con los mayores niveles de discapacidad en 1996 eran Finlandia $(22,9 \%)$ y Reino Unido $(18.8 \%)$ y aquellos con los menores Italia $(7,8 \%)$ y Bélgica $(8,2 \%)$. En España la tasa se situaba en el $9,9 \%$, inferior a la media Europea $(14,8 \%)$.

\subsection{Situación actual}

Para dar respuesta a las preguntas ¿quiénes son las personas discapacitadas? ¿cuáles son sus principales características demográficas?, es necesario tener en cuenta dos aspectos. En primer lugar, debido a que el marco de referencia de esta investigación es el mercado de trabajo, se limitará el estudio a la población con edades comprendidas entre los 16 y 64 años. En segundo lugar, se analiza la situación utilizando el último año disponible hasta la fecha en el PHOGUE, es decir, el año 1998 para de este modo tener los datos lo más actualizados posible. $Y$ en tercer lugar, hay que tener en cuenta que el colectivo formado por personas discapacitadas es tremendamente heterogéneo, formado por personas con diferentes grados y tipos de discapacidad. Es posible hablar de discapacitados congénitos o con discapacidad adquirida, físicos o psíquicos, temporales o permanentes, capacitados o incapacitados para trabajar, etc. Esto hace que el análisis se pueda ir ramificando y complicando a medida que se vaya descendiendo y eliminando paulatinamente esta heterogeneidad. En la mayoría de los estudios existentes el análisis desciende hasta el tipo de discapacidad y a partir de aquí se combina con diferentes variables socioeconómicas relevantes (edad, sexo, nivel educativo, etc.). Debido a que en el PHOGUE no es posible identificar qué tipo de discapacidad padece el individuo, se ha diferenciado a aquellas personas que tienen una discapacidad severa de aquellas con una discapacidad moderada (a partir de la pregunta PHOO3) ${ }^{n}$.

5. Este grupo de expertos depende de la Dirección General de Empleo y Asuntos Sociales de la Comisión Europea y tiene como objetivo recopilar y analizar la información disponible sobre la situación de las personas discapacitadas en la Unión Europea. También, se configura como un marco de referencia para la propuesta y elaboración de políticas públicas que favorezcan la integración de este colectivo.

6 Aunque la clasificación de las personas discapacitadas entre aquellas con una discapacidad severa y aquellas con una discapacidad moderada no se ajusta a la clasificación realizada por la OIS se ha considerado relevante para, en cierta manera, tener en cuenta la heterogeneidad del colectivo de las personas discapacitadas. Esta diferenciación se ha utilizado en otros estudios como, por el ejemplo, el realizado por la OCDE (2003). 
Según los datos del cuadro 1 , si se comparan las características de las personas discapacitadas con las de las no discapacitadas se observa una distribución por edades completamente distinta. Existe una evidente y clara relación entre edad y discapacidad. La probabilidad de tener una discapacidad aumenta con la edad, especialmente a partir de los 45 años. Por ejemplo, el $41,31 \%$ de las personas con edades comprendidas entre 55 y 64 años padece una discapacidad. En cambio, este porcentaje cae hasta el 8,19\% para aquellas personas con edades comprendidas entre los $16 \mathrm{y}$ 24 años. Por grado de discapacidad, para aquellos individuos con una discapacidad severa se aprecia una mayor concentración en los intervalos de edad más elevados en relación a los que presentan una discapacidad moderada.

La presencia de un mayor número de personas discapacitadas en los tramos más elevados de edad está asociada al hecho de que el estado de salud de las personas se deteriora con la edad, adquiriendo nuevas deficiencias y problemas de salud. También, existe un factor generacional que hay que tener en cuenta. Las personas más jóvenes han disfrutado de mejores condiciones de salud, seguridad en el trabajo, servicios asistenciales, etć. que las personas de generaciones más antiguas. Este hecho reduce la probabilidad de que las personas más jóvenes sean o lleguen a ser discapacitadas. En contra de esta opinión, algunos autores también han apuntado que en la sociedad actual existe un mayor estrés y ritmo de vida, especialmente para los más jóvenes, $\mathrm{y}$, por tanto, un mayor riesgo a sufrir éstos enferrnedades mentales.

Por sexos, las diferencias entre personas discapacitadas y no discapacitadas son insignificantes, siendo la presencia de ambos sexos igualitaria. Sin embargo, si se aprecian diferencias importantes dentro del colectivo de las personas discapacitadas según el grado de la discapacidad. Existe un mayor porcentaje de hombres que de mujeres que padecen una discapacidad severa, un $54,99 \%$ frente a un $45,01 \%$. Los resultados por sexos se invierten cuando la discapacidad es moderada, siendo ahora en un $53,51 \%$ de los casos mujeres.

\section{Cuadro 1}

Características sociodemográficas de las personas discapacitadas y no discapacitadas (en porcentaje). Año 1998.

\section{Edad}

$16-24$

8,19

$25-34$

9,35

6,11

9,18

17,05

16,20

11,50

22,85

$35-44$

17,05

9,63

26,07

18,41

21,81 


$\begin{array}{lllll}45-54 & 24,10 & 25,78 & 21,43 & 17,10 \\ 55-64 & 41,31 & 42,73 & 39,04 & 12,17 \\ & & & & \\ \text { Sexo } & & & & \\ \text { Mujer } & 50,23 & 45,01 & 53,51 & 50,32 \\ \text { Varón } & 49,77 & 54,99 & 46,49 & 49,68\end{array}$

Nivel educativo

$\begin{array}{lrrrr}\text { Analfabetos } & 27,17 & 40,49 & 18,80 & 6,00 \\ \text { Primaria } & 48,99 & 42,01 & 53,39 & 26,42 \\ \text { Secundaria } 1^{\circ} \text { ciclo } & 15,64 & 11,98 & 17,94 & 30,61 \\ \text { Secundaria } 2^{\circ} \text { ciclo } & 5,64 & 3,44 & 7,03 & 21,77 \\ \text { Universitarios } & 2,55 & 2,08 & 2,84 & 15,20\end{array}$

\section{Región de residencia}

$\begin{array}{lrrrr}\text { Noroeste } & 12,47 & 11,72 & 12,93 & 12,31 \\ \text { Noreste } & 7,47 & 6,82 & 7,88 & 10,31 \\ \text { Madrid } & 8,47 & 4,60 & 10,89 & 16,28 \\ \text { Centro } & 18,31 & 24,94 & 14,17 & 12,28 \\ \text { Este } & 27,37 & 25,81 & 28,35 & 25,27 \\ \text { Sur } & 20,78 & 22,51 & 19,70 & 20,15 \\ \text { Canarias } & 5,13 & 3,61 & 6,08 & 3,41\end{array}$

Fuente: Panel de Hogares de la Unión Europea (PHOGUE). Año 1998.

Uno de los principales factores que influyen en la integración laboral de las personas discapacitadas ( $\mathrm{y}$ también no discapacitadas) es el nivel educativo del individuo. El cuadro 1 muestra que las personas discapacitadas poseen unos niveles educativos muy bajos comparado con las personas no discapacitadas. Tres de cada cuatro personas discapacitadas son analfabetas o tienen estudios primarios. Si la discapacidad es severa entonces el porcentaje de individuos situados en los dos niveles educativos más bajos aumenta hasta el $82,5 \%$. Las diferencias con el colectivo formado por las personas no discapacitadas son enormes. Sirva de ejemplo el hecho de que sólo un $2,55 \%$ de los discapacitados son universitarios, mientras que para los no discapacitados este porcentaje se eleva hasta el $15,20 \%{ }^{7}$.

7. Con respecto a las personas discapacitadas que se encuentran estudiando una carrera universitaria o ya la han finalizado hay que tener en cuenta diversos aspectos. De acuerdo con la Ley de Integración Social de Minusválidos de 1982, que regula el acceso a la educación y cuyo desarrollo corresponde a las comunidades autónomas, todos los centros, entre ellos los universitarios, tienen la obligación de reservar el $3 \%$ de sus plazas a las personas discapacitadas. La realidad actual demuestra estas plazas nunca se cubren. Aun- 
Además, aunque en el cuadro no se muestra, la precariedad educativa de las personas discapacitadas es ligeramente superior para el caso de las mujeres que para el caso de los hombres.

Los bajos niveles educativos de las personas discapacitadas pueden proceder de tres efectos diferentes. En primer lugar, existe un "efecto edad". Las generaciones mayores son las menos educadas. De este modo, la positiva correlación entre edad y discapacidad, hace que exista una correlación entre un menor nivel educativo y discapacidad. En segundo lugar, existe un "efecto riesgo". Las personas con un nivel educativo bajo tienden a tener peores trabajos que implican en muchos casos mayores riesgos y accidentes de trabajo, y, por tanto, llegar a convertirse en personas discapacitadas. Y en tercer lugar, se puede hablar de un "efecto inverso", es decir, el efecto que tiene la discapacidad en el nivel educativo de la personá.

Las personas con determinados tipos de discapacidad severa se encuentran con barreras y menores oportunidades de obtener un mayor nivel educativo. Según la "Encuesta sobre Discapacidades, Deficiencias y Estado de Salud" (EDDES) realizada en 1999, los mayores niveles educativos se dan entre las personas con discapacidades sensoriales. Por el contrario, los niveles educativos más bajos se concentran en las discapacidades de la conducta, de la comunicación, del aprendizaje, y del cuidado de sí mismas. Las discapacidades que afectan a la movilidad del individuo se sitúan en la banda intermedia.

La mayor o menor presencia de personas discapacitadas no es homogénea a lo largo de todo el territorio nacional. Las comunidades autónomas situadas en el Este (Cataluña, Comunidad Valenciana y Baleares) son las que agrupan un mayor número de personas discapacitadas, seguidas de las comunidades sureñas (Andalucía, Murcia, Ceuta y Melilla).

\section{DisCAPACIDAD Y EMPLEO}

Según el cuadro 2, existen importantes diferencias con respecto a la situación laboral de las personas discapacitadas y no discapacitadas. Algo menos del 35\% de las personas discapacitadas eran activas en 1998, mientras que este porcentaje alcanzaba casi el $66 \%$ para las personas no discapacitadas. Esto significa que un $65,13 \%$ de los discapacitados eran inactivos (34,09\% para los no discapacitados). El 7,65\%

que la matricula para estas personas es gratuita en muchos comunidades autónomas, como. Murcia y Andalucía, el problema que existe es que muchos discapacitados optan por no decir que lo son al pedir plaza en la universidad, ya sea porque ya tienen una ayuda o porque no la necesitan, Este problema dificulta la identificación de las personas discapacitadas por parte de la universidad (colectivo oculto) y otros agentes económicos y sociales interesados. Este colectivo oculto también puede tener influeincia en las cifras obtenidas del PHOGUE, de tal modo que las cifras obtenidas puedan estar infraestimadas. 
de las personas discapacitadas estaba parada y el $27,22 \%$ trabajando, cifra esta última muy inferior a la de las personas no discapacitadas. Por grado de discapacidad también se aprecian diferencias. El porcentaje de personas con una discapacidad severa que está trabajando es aproximadamente la mitad del de las personas con una discapacidad moderada y un tercio del de las personas no discapacitadas. Además, el porcentaje de activos es sólo del 21,49\%, estando la gran mayoría de estas personas con una discapacidad severa situadas en la inactividad.

\section{Cuadro 2}

Situación laboral de las personas discapacitadas y no discapacitadas (en porcentaje). Año $1998^{8}$.

DISCAPACITADOS

TODOS SEVERA

NO DISCAPACITADOS MODERADA

\begin{tabular}{lrrrr} 
Activos & 34,87 & 21,49 & 43,14 & 65,91 \\
Trabajando & 27,22 & 17,24 & 33,48 & 53,19 \\
Parado & 7,65 & 4,45 & 9,66 & 12,72 \\
Inactivos & 65,13 & 78,31 & 56,85 & 34,09 \\
\hline TOTAL & 100,00 & 100,00 & 100,00 & 100,00 \\
\hline
\end{tabular}

Fuente: PHOGUE. Año 1998.

En el cuadro 3 se recogen las tasa de paro y actividad para ambos colectivos. También se ha calculado el cociente entre la tasa de paro o actividad de las personas discapacitadas (distinguiendo de nuevo entre severa y moderada) y la de las personas no discapacitadas. La tasa de paro de las personas discapacitadas es del $21,94 \%$ y superior a la de las personas no discapacitadas $(19,3 \%)$ en un $14 \%$. La mayor diferencia se encuentra en las tasas de actividad como se ha apuntado anteriormente en el cuadro 2 . La tasa de actividad de las personas discapacitadas es del $34,87 \%$, la cual representa sólo un $53 \%$ de la de las personas no discapacitadas. Por grado de discapacidad, los resultados también muestran diferencias especialmente en las tasas de actividad a favor de las personas con una discapacidad moderada (casi el doble) en comparación con las personas con una discapacidad severa.

8. Para determinar la situación laboral del individuo se ha utilizado la variable PE003 del cuestionario la cuál clasifica a los individuos siguiendo los criterios utilizados en la Encuesta de Población Activa (EPA). 


\section{Cuadro 3}

Tasas de paro y actividad de las personas discapacitadas y no discapacitadas.

Año 1998.

DISCAPACITADOS

NO DISCAPACITADOS

TODOS SEVERA MODERADA

$\begin{array}{lrrrr}\text { Tasa de paro } & 21,94 & 20,52 & 22,39 & 19,30 \\ \text { Ratio Disca/No disca } & 1,14 & 1,06 & 1,16 & \\ \text { Tasa de actividad } & 34,87 & 21,69 & 43,15 & 65,91 \\ \text { Ratio Disca/No disca } & 0,53 & 0,33 & 0,65 & \end{array}$

Fuente: PHOGUE. Año 1998

Si se comparan las tasas de paro y actividad calculadas con el PHOGUE para el año 1998 y las obtenidas en la EDDES de 1999 se aprecian diferencias ${ }^{9}$. La razón de estas diferencias procede de las diferentes definiciones de la discapacidad y la población incluida, las técnicas usadas, el momento temporal y el objetivo final de cada encuesta. El PHOGUE no es una encuesta diseñada específicamente para estudiar al colectivo formado por las personas discapacitadas en España, mientras que la EDDES si ha sido diseñada con ese objetivo. Estas consideraciones provoca que los resultados obtenidos con el PHOGUE sean diferentes de los recogidos en la EDDES.

Debido a que el objetivo final del estudio es analizar la integración laboral de las personas discapacitadas se ha considerado relevante llevar a cabo un análisis más orientado y detallado de la población discapacitada que está actualmente trabajando. Para ello, en primer lugar se comparan las características socio demográficas de las personas discapacitadas empleadas con las características del total de personas discapacitadas. En segundo lugar, se comparan las características de las personas discapacitadas empleadas con las de las personas no discapacitadas que trabajan. Y en tercer lugar, se analizan las características laborales del puesto de trabajo de las personas discapacitadas y las de las no discapacitadas.

\footnotetext{
9 Según los resultados de la "Encuesta sobre Discapacidades, Deficiencias y Estado de Salud" (EDDES) realizada en 1999, la tasa de paro de las personas discapacitadas fue del $25,8 \%$ en el momento de realizar la encuesta (segundo trimestre de 1999). Para ese mismo periodo, la "Encuesta de Pablación Activa" (EPA) reflejaba una tasa de paro para la población general del $15,7 \%$. Con respecto a la tasa de actividad de las personas con discapacidades, ésta es inferior a la de la población general (32,3\% en comparación con el 50\%), tasas definidas sobre la población de 16 y más años (a diferencia de la definida en este trabajo, donde se utiliza la población entre 16 y 64 años).
} 


\section{Cuadro 4}

Características sociodemográficas de las personas discapacitadas. Total y empleadas (en porcentaje). Año 1998.

\begin{tabular}{|c|c|c|c|c|c|c|c|c|}
\hline \multicolumn{9}{|c|}{ DISCAPACITADOS } \\
\hline & \multicolumn{2}{|c|}{ TODOS } & \multicolumn{2}{|c|}{ SEVERA } & \multicolumn{2}{|c|}{ MODERADA } & \multicolumn{2}{|c|}{$\begin{array}{c}\text { No } \\
\text { DISCAPACITADOS }\end{array}$} \\
\hline & \multicolumn{2}{|c|}{ Total Trabajando } & Total & Trabajando & Total & Trabajandd & \multicolumn{2}{|c|}{ Total Trabajando } \\
\hline \multicolumn{9}{|l|}{ Edad } \\
\hline $16-24$ & 8,19 & 6,51 & 6,11 & 0,91 & 11.50 & 8,32 & 22,85 & 11,64 \\
\hline $25-34$ & 9,35 & 14,58 & 9,18 & 10,26 & 9.63 & 15,98 & 26,07 & 31,54 \\
\hline $35-44$ & 17,05 & 27,66 & 16,20 & 43,86 & 18,41 & 22.42 & 21,81 & 28,30 \\
\hline $45-54$ & 24,10 & 28,78 & 25,78 & 18,53 & 21,43 & 32.10 & 17,10 & 20,40 \\
\hline $55-64$ & 41,31 & 22,46 & 42,73 & 26,43 & 39,04 & 21,18 & 12,17 & 8,13 \\
\hline \multicolumn{9}{|l|}{ Sexo } \\
\hline Mujer & 50,23 & 35,64 & 45,01 & 23,17 & 53.51 & 39,68 & 50,32 & 35,18 \\
\hline Varón & 49,77 & 64,36 & 54,99 & 76,83 & 46,49 & 60,32 & 49,68 & 64,82 \\
\hline \multicolumn{9}{|c|}{ Nivel educativo } \\
\hline Analfabetos & 27,17 & 15,73 & 40,49 & 38,63 & 18,80 & 8.30 & 6,00 & 3,11 \\
\hline Primaria & 48,99 & 49,12 & 42,01 & 41,72 & 53,39 & 51,52 & 26,42 & 25,19 \\
\hline Secundaria $1^{\circ}$ ciclo & 15,64 & 18,02 & 11,98 & 11,86 & 17,94 & 20,02 & 30,61 & 28,93 \\
\hline Secundaria $2^{\circ}$ ciclo & 5,64 & 12,40 & 3,44 & 6,40 & 7,03 & 14,34 & 21,77 & 21,30 \\
\hline Universitarios & 2,55 & 4,73 & 2,08 & 1,39 & 2,84 & 5,81 & 15,20 & 21,46 \\
\hline \multicolumn{9}{|c|}{ Región de residencia } \\
\hline Noroeste & 12,47 & 11,14 & 11,72 & 4,88 & 12,93 & 13,17 & 12,31 & 11,99 \\
\hline Noreste & 7,47 & 6,83 & 6,82 & 7,88 & 7,88 & 6,49 & 10,31 & 10,72 \\
\hline Madrid & 8,47 & 12,56 & 4,60 & 8,59 & 10,89 & 13,85 & 16,28 & 16,86 \\
\hline Centro & 18,31 & 16,40 & 24,94 & 32,27 & 14,17 & 11,27 & 12,28 & 10,78 \\
\hline Este & 27,37 & 30,87 & 25,81 & 32,62 & 28,35 & 30,30 & 25,27 & 29,64 \\
\hline Sur & 20,78 & 19,05 & 22,51 & 11,80 & 19,70 & 21,40 & 20,15 & 16,81 \\
\hline Canarias & 5,13 & 3,14 & 3,61 & 1,96 & 6,08 & 3,52 & 3,41 & 3,20 \\
\hline
\end{tabular}


En el primer caso. los resultados obtenidos reflejan que las personas discapacitadas que trabajan son más jóvenes, frecuentemente hombres y con un mayor nivel educativo con respecto al total de la población discapacitada (cuadro 4). Por región de residencia no se aprecian diferencias significativas. Con respecto al nivel educativo, el porcentaje de discapacitados trabajando con estudios de secundaria $2^{\circ}$ ciclo aumenta considerablemente. al igual que el porcentaje de licenciados, aunque en menor medida. Cuando se comparan los resultados obtenidos según el grado de discapacidad. cabe mencionar que las personas con una discapacidad severa que trabajan suelen tener una edad comprendida entre los 35 y 4 t años. dos terceras partes son hombres. la inmensa mayoria son analfahetos o poseen estudios primarios y se concentran especialmente en las regiones del centro y este del territorio español.

En el segundo caso. cuando se comparan las caracteristicas de las personas discapacitadas y no discapacitadas que están trabajando se obtiene un resultado muy diferente al anterior. La persona discapacitada trabajadora tiene mayor edad y está bastante menos educada que la persona no discapacitada. No se aprecian diferencias de género significativas entre amboss colectivos. Las mayores diferencias educativas se dan en los niveles educativos superiores. Por grados de discapacidad. las personas con una discapacidad severa que trabajan son muchos más mayores. con un claro predominio de los hombres y con grandes diferencias educativas a favor de las personas que no son discapacitadas. Por ejemplo, sólo un $1.39 \%$ de las personas con una discapacidad severa posec un título universitario mientras que este porcentaje sube hasta el $21,46^{1 / \%}$ para el colectivo de las personas que no padecen una discapacidad. Por regiones, se aprecian patrones similares para todos los casos, aunque el peso de cada región varia según el colectivo que se esté analizando.

Con respecto a las caracteristicas laborales del puesto de trabajo que ocupa la persona discapacitada en comparación con el que ocupa la persona no discapacita$\mathrm{da}$, no se aprecian diferencias significativas en cuanto al nivel de autoempleo/empleo por cuenta ajena (cuadro 5). Sin embargo, cuando la persona tiene una discapacidad severa el porcentaje de autónomos se reduce casi a la mitad. Existe una mayor presencia de personas discapacitadas trabajando en el sector privado con respecto a las no discapacitadas aunque la diferencias no son muy elevadas. Cuando la persona tiene una discapacidad severa el porcentaje de éstas que trabajan en el sector privado aumenta ligeramente.

Por sector de actividad, para todos los colectivos considerados el sector servicios es el que absorbe al mayor número de personas que están trabajando. Comparativamente, las diferencias no son muy significativas, aunque cabe mencionar la menor presencia de personas con una discapacidad severa en el sector agrícola con respecto al total de personas discapacitadas. La utilización de contratos indefinidos es mayor para las personas no discapacitadas que para las discapacitadas, excepto en el caso de que la persona tenga una discapacidad severa. La utilización de otro 
tipo de contratos, entre ellos los dirigidos específicamente al colectivo de las personas discapacitadas, son utilizados por personas con una discapacidad moderada en un $10,32 \%$ de los casos.

\section{Cuadro 5}

Características laborales del puesto de trabajo de las personas discapacitadas y no discapacitadas (en porcentaje). Año 1998.

\begin{tabular}{|c|c|c|c|c|}
\hline \multicolumn{4}{|c|}{ DISCAPACITADOS } & \multirow[b]{2}{*}{\begin{tabular}{|c|} 
No \\
DISCAPACITADOS
\end{tabular}} \\
\hline & TODOS & SEVERA & MODERADA & \\
\hline \multicolumn{5}{|l|}{ Tipo de empleo } \\
\hline Asalariado & 76,67 & 87,79 & 73,02 & 78,88 \\
\hline Autónomo & 23,33 & 12,21 & 26,98 & 21,12 \\
\hline \multicolumn{5}{|l|}{ Tipo de sector } \\
\hline Privado & 90,01 & 93,06 & 89,11 & 81,96 \\
\hline Público & 9,99 & 6,94 & 10,89 & 18,04 \\
\hline \multicolumn{5}{|l|}{ Sector actividad } \\
\hline Agricultura & 11,66 & 7,15 & 13,12 & 7,27 \\
\hline Industria & 29,29 & 32,53 & 28,25 & 29,04 \\
\hline Servicios & 59,05 & 60,32 & 58,64 & 63,68 \\
\hline \multicolumn{5}{|l|}{ Tipo de contrato } \\
\hline Indefinido & 59,84 & 81,35 & 51,18 & 66,27 \\
\hline Eventual o temporal & 30,21 & 18,65 & 34,87 & 27,57 \\
\hline No tiene contrato & 2,59 & 0,00 & 3,63 & 3,51 \\
\hline Otros & 7,36 & 0,00 & 10,32 & 2,66 \\
\hline \multicolumn{5}{|c|}{ Tamaño de la empresa } \\
\hline De 1 a 19 & 62,18 & 61,23 & 62,51 & 48,30 \\
\hline De 20 a 49 & 14,87 & 7,60 & 17,33 & 15,81 \\
\hline De 50 a 99 & 7,50 & 10,31 & 6,55 & 9,63 \\
\hline De 100 o más & 15,45 & 20,86 & 13,61 & 26,27 \\
\hline \multicolumn{5}{|l|}{ Horas de trabajo } \\
\hline Menos de 15 & 5,56 & 9,30 & 4,38 & 3,68 \\
\hline Entre 15 y 30 horas & 15,38 & 8,73 & 17,47 & 9,69 \\
\hline Más de 30 horas & 79,07 & 81,97 & 78,15 & 86,62 \\
\hline
\end{tabular}


Por tamaño de empresa se aprecian diferencias significativas en los extremos. Las personas discapacitadas trabajan sobre todo en empresas compuestas por un número de trabajadores situade entre 1 y 19 . Este porcentaje se sitúa en el $62,18 \%$ de media, superior al registrado para las personas no discapacitadas que es del $48,30 \%$. Si a este primer tramo se le une el segundo tramo considerado, es decir, el tramo de 20 a 29 trabajadores, el porcentaje de personas discapacitadas que trabajan en empresas con menos de 50 trabajadores alcanza la cifra del $77,05 \%$, muy superior al $64,31 \%$ del de las personas no discapacitadas. La mayor presencia de personas discapacitadas en empresas con menos de 50 trabajadores está relacionada con la cuota de reserva del $2 \%$ para las personas discapacitadas que establece la legislación española, pero que según muchos agentes económicos y sociales no se está cumpliendo en la práctica, incluso por la propia administración pública la cuál está obligada a reservar un $3 \%$ de los ofertas de empleo a las personas discapacitadas. Además, hay que tener en cuenta que la gran mayoría de las empresa que llevan a cabo su actividad empresarial en España son PYMES o micro empresas, lo que provoca que la gran mayoría de las ofertas de trabajo existentes destinadas tanto a personas discapacitadas como no discapacitadas provengan de este tipo de empresas.

También se aprecian diferencias importantes en cuanto a la jornada de trabajo que realizan las personas discapacitadas. Las personas discapacitadas trabajan con mayor frecuencia jornadas a tiempo parcial (30 o menos horas de trabajo a la semana) en comparación con las personas no discapacitadas. Estas preferencias por la jornada a tiempo parcial son realizadas sobre todo por personas que tienen una discapacidad moderada, en cuyo caso el porcentaje de personas con una jornada a tiempo parcial se sitúa en el $21,85 \%$. Este resultado viene motivado por el hecho de que según el grado de la discapacidad que padece el individuo limite el número máximo de horas disponibles que puede dedicar en el mercado de trabajo, y de este modo preferir trabajar una jornada a tiempo parcial antes que una jornada a tiempo completo. Desde el punto de vista de las políticas públicas, es recomendable que una persona discapacitada participe y encuentre un empleo, aunque sea a tiempo parcial, a una persona que se encuentra anclada en una situación de inactividad y con muchas dificultades de salir de ella.

\section{ConClusiones}

A partir de los resultados obtenidos, se aprecia que uno de los principales obstáculos para la integración laboral de las personas discapacitadas se sitúa en la primera fase del proceso de inserción. Es decir, en pasar de la inactividad a la actividad. Para que una persona encuentre trabajo son necesarias dos decisiones. Primero, decidir buscar trabajo, es decir, participar en el mercado de trabajo. Y segundo, 
encontrar un trabajo que se ajuste a lo que busca el individuo. Para el caso particular de las personas discapacitadas el primer paso, el participar en el mercado de trabajo, constituye una de las mayores razones de peso para explicar los bajos niveles de actividad y los elevados niveles de desempleo existentes en la actualidad.

Las personas discapacitadas empleadas poseen una mayor edad, están menos educadas, y ocupan puestos de trabajo con características inferiores (por ejemplo, ocupaciones poco cualificadas y con contratos más precarios) en comparación con las características de las personas no discapacitadas empleadas. El ser discapacitado es ya en sí una discriminación a nivel social y laboral que se ve reflejado en los indicadores básicos del mercado de trabajo. Es sabido que en España el número de personas con discapacidad incorporadas al mercado de trabajo se sitúa en tasas muy inferiores a las de la población en general, quedando pendiente la enorme tarea de atender las necesidades reales de quiénes están en edad laboral y tratan infructuosamente de participar personal y económicamente en nuestra sociedad a través de su trabajo.

Un reciente informe de la OCDE (2003), propone para mejorar la situación laboral y social de la persona discapacitada llevar a cabo reformas basadas en cinco puntos clave: a) Reconocer el estado de discapacidad independientemente de la situación laboral o económica del individuo; b) incentivar a la personas discapacitadas a participar en el mercado de trabajo; c) reestructurar los sistemas públicos de ayudas para que, sobre todo, no penalice a la persona discapacitada en el caso de encontrar y aceptar un trabajo; d) introducir una cultura de obligaciones mutuas donde la persona discapacitada que reciba ayuda esté condicionada a participar en el empleo, programas de rehabilitación vocacional y formación y otras medidas de integración; y e) involucrar a los empresarios en el proceso ya que son ellos los que en última instancia demanda trabajadores. Además, el informe apunta que en muchos países los programas de integración a través de la rehabilitación vocacional y el empleo protegido son exclusivamente ofrecidos a personas con 45 o menos años, lo cuál explica el por qué muchas de las personas mayores se encuentran anclados en las sistemas públicos de ayuda a la discapacidad. También y muy relacionado con los resultados obtenidos en este trabajo se critica el hecho de que muchas políticas tienden más a favorecer a las personas discapacitadas ya empleadas que a las personas que se encuentran fuera del mercado de trabajo. 


\section{BIBLIOGRAFÍA}

Baldwin, M. y Johnson. W.G. (1994), "Labor market discrimination against men with disabilities", Journal of Human Resources, XXIX (31), 865-887.

Bardasi, E., Jenkins S. y Rigg J. (2000), "Disability, work and income: a British perspectiva", Documento de Trabajo del Institute for Social and Economic Research, Universidad de Essex, ${ }^{\circ}$ 2000/36.

Berkowitz, M. y Johnson, W. (1974), "Health and labor force participation", Journal of Human Resources, 9, 117-128.

Brunel (2001), Discussion Paper for Workshop Feb $5^{\text {th }}$ - Feb $7^{\text {b }} 2001$.

Burchardt, T. (2000), "The dynamics of being disabled", Journal of Social Policy, 29, 4, 645-668.

Consejo Económico y Social (1995), Informe sobre la situación del empleo de las personas con discapacidad y propuestas para su reactivación, monografía.

Jenkins, S. y Rigg, J. A. (2003), "Disability and disadvantage: selection, onset and duration effects", ISER Working Papers, 18.

Kidd, M. P., Sloane, P. J. y Ferko, I. (2000), " Disability and the labour market: an analysis of British males", Journal of Health Economics, 19, 961-981.

Malo, M. A. y García, C. (2001), Employment and disability in the European Community Housebold Panel: A first approach, monografía.

OCDE (2003), Transforming Disability into Ability: Policies to Promote Work and Income Security for Disabled People, monografía.

OCDE (2003), "Disability programmes in need of reform", Policy Brief, march.

O'Donnell, O. (1998), "The effect of disability on employment allowing for work incapacity", Documento de trabajo de Universidad de Kent, 98/13.

Salkever, D.S. y Sorkin, A. (2003), The Economics of Disability, Jai Press inc, Connecticut. 